\title{
A Lunar Liquid Mirror Telescope (LLMT) for deep-field infrared observations near the lunar pole
}

\author{
Roger Angel ${ }^{\mathrm{a}}$, Dan Eisenstein ${ }^{\mathrm{a}}$, Suresh Sivanandam ${ }^{\mathrm{a},}$ Simon P. Worden ${ }^{\mathrm{a}}$, Jim Burge ${ }^{\mathrm{b}}$, Ermanno \\ Borra $^{\mathrm{c}}$, Clément Gosselin ${ }^{\mathrm{c}}$, Omar Seddiki ${ }^{\mathrm{c}}$, Paul Hickson ${ }^{\mathrm{d}}$, Ki Bui Ma ${ }^{\mathrm{e}}$, Bernard Foing ${ }^{\mathrm{f}}$, Jean-Luc \\ Josset $^{\mathrm{g}}$, Simon Thibault ${ }^{\mathrm{h}}$, Paul Van Susante ${ }^{\mathrm{i}}$ \\ ${ }^{\mathrm{a}}$ Steward Observatory, The University of Arizona, 933 N. Cherry Avenue, Tucson, AZ USA 85751 \\ ${ }^{\mathrm{b}}$ Optical Sciences Center, The University of Arizona, Tucson,1630 E. University Blvd., Tucson, AZ \\ USA 85721 \\ ${ }^{\mathrm{c}}$ Université Laval, Faculté des sciences et de génie, Pavillon Alexandre-Vachon, Québec Canada \\ G1K7P4 \\ ${ }^{\mathrm{d}}$ Department of Physics \& Astronomy, University of British Columbia, 6224 Agricultural Road \\ Vancouver, B.C,. V6T 1Z1, Canada \\ e Texas Center for Superconductivity, University of Houston, Houston TX USA 77204 \\ ${ }^{\mathrm{f}}$ Chief Scientist, ESA/ESTEC/SCI-S, postbus 299 Noordwijk 2200 AG Noordwijk, The \\ Netherlands \\ ${ }^{\mathrm{g}}$ Space-X Space Exploration Institute, 1 rue Jaquet-Droz, CH-2007 Neuchâtel, Switzerland 2020 \\ ${ }^{\mathrm{h}}$ Immervision, 2020 University Avenue, Montreal, Québec, Canada, H3A 2A5 \\ ${ }^{\mathrm{i}}$ Engineering, Colorado School of Mines, 1500 Illinois St, Golden, CO USA 80401
}

\begin{abstract}
We have studied the feasibility and scientific potential of a $20-100 \mathrm{~m}$ aperture astronomical telescope at the lunar pole, with its primary mirror made of spinning liquid at less than $100 \mathrm{~K}$. Such a telescope, equipped with imaging and multiplexed spectroscopic instruments for a deep infrared survey, would be revolutionary in its power to study the distant universe, including the formation of the first stars and their assembly into galaxies. The LLMT could be used to follow up discoveries made with the $6 \mathrm{~m}$ James Webb Space Telescope, with more detailed images and spectroscopic studies, as well as to detect objects 100 times fainter, such as the first, high-red shift stars in the early universe. Our preliminary analysis based on SMART-1 AMIE images shows ridges and crater rims within $0.5^{\circ}$ of the North Pole are illuminated for at least some sun angles during lunar winter. Locations near these points may prove to be ideal for the LLMT. Lunar dust deposited on the optics or in a thin atmosphere could be problematic. An in-situ site survey appears necessary to resolve the dust questions.
\end{abstract}

Keywords: Lunar telescopes, rotating cryogenic liquid mirrors, early universe

\section{INTRODUCTION}

Our lunar liquid mirror telescope concept is aimed at furthering our understanding of the early universe. This has recently been revolutionized by deep optical fields imaged first with the Hubble Space Telescope (HST), and followed up by observations by other telescopes across the electromagnetic spectrum, including the Spitzer infrared telescope, and by spectroscopic analysis with 6-10 m ground-based telescopes. In the next 10-20 years Hubble's successor, the $6 \mathrm{~m}$ infrared James Webb Space Telescope (JWST) will work in tandem with 20-30 m class ground telescopes now being planned, such as the Thirty Meter Telescope (TMT) and Giant Magellan Telescope (GMT). However, for high red shift

Space Telescopes and Instrumentation I: Optical, Infrared, and Millimeter, edited by John C. Mather, Howard A. MacEwen, Mattheus W.M. de Graauw,

Proc. of SPIE Vol. 6265, 62651U, (2006) - 0277-786X/06/\$15 - doi: 10.1117/12.669994

Proc. of SPIE Vol. 626562651 U-1 
studies of the early universe, it will no longer be possible to use larger ground apertures to follow up the very high red shifted faint objects found by the JWST, because of high atmospheric and thermal background in the infrared. To learn more about much deeper fields, a very cold space telescope such as the one we propose will be required, with aperture of 20-100 meters diameter and capable of integrations of days to years with Hubble quality imaging.

The Moon is potentially an ideal location for such ultra-deep observations over the full electromagnetic spectrum, completely free from atmospheric absorption and distortion. Ultra deep fields can be chosen anywhere in the sky that is not compromised by dust in our own solar system or galaxy. From the Moon it is convenient to observe fields at or near the ecliptic poles, because the spin axis of the moon points always close to this direction. This allows a great simplification, in that telescopes do not have to be controlled to track stars around the sky. We can take advantage of the Moon as a stable platform to keep a telescope pointed constantly out along the Moon's spin axis. The field will slowly move (because of the Moon's 18.6 year precession) around the ecliptic pole in a circular path of $3^{\circ}$ diameter, at $0.5^{\circ}$ per year. An advantage of the ecliptic poles is that they are the two regions on the sky where the infrared sky background from zodiacal dust is minimized, and are thus preferred in any case for deep infrared observations. The lunar telescope one would likely choose to build first would be to survey primarily the infrared in the wavelength range $1-10 \mu \mathrm{m}$, for the following reasons:

1) It is a spectral region of very high scientific interest, particularly for the study of the early universe. The radiation of the first stars emitted primarily at rest wavelengths from $0.1-1$ micron is red shifted by around a factor 10 to this region.

2) The zodiacal sky background is lowest in the $2-5 \mu \mathrm{m}$ spectral region, allowing for extremely deep images. The 6-m James Webb Space Telescope (JWST), the successor to HST, is focused on the same spectral region for the above reasons. JWST will obtain the first really deep images of the high red shift universe. We should be clear that while a fixed axis lunar telescope would be exceptional for early universe studies, because there is an abundance of high red shift targets is any direction, it is not useful for rare targets (such as nearby extra solar planets) where we need access the whole sky. For the latter the best location might well be a gravity-free environment far from the warm Earth, such as L2. But the assembly and maintenance of a diffraction- limited 20-100 $\mathrm{m}$ diameter cryogenic telescope at L2, a million miles from earth, may well remain beyond reach for the foreseeable future. But the same aperture dedicated to a deep survey may well be feasible on one of the Moon's poles, by taking advantage of a trick to make a very high quality liquid mirror with the aid of gravity.

Primary mirrors made as a spinning dish of liquid in a gravity field represent completely different technology, with the potential to achieve very large size and high optical quality for an affordable system. Liquid mirrors take on a smooth and precise surface automatically, and when rotating in a gravitational field takes the parabolic figure needed for a telescope primary. Mercury has been used to make such inexpensive mirrors with excellent surface quality. The technology is young but its performance is well documented by laboratory tests (Girard \& Borra $1997^{1}$, Tremblay \& Borra $2000^{2}$ ) as well as by observations (Sica et al. $1995^{3}$, Hickson \& Mulrooney $1998^{4}$, Cabanac, Borra, \& Beauchemin $1998^{5}$ ). Zenith-pointing telescope mirrors of liquid mercury have been made at very low cost up to 6-m diameter Large Zenith Telescope Survey, spinning at a few revolutions per minute in the 1-g gravity field of Earth (www.astro.ubc.ca/lmt/lzt), see also Cabanac, Hickson and de Lapparent, $2002^{6}$ ). Indeed, it appears that liquid mirror telescopes such as the 6-m diameter system built by Paul Hickson and his collaborators at the University of British Columbia can be constructed for a few percent the cost of corresponding conventional telescope designs of a similar aperture (Hickson and Lanzetta, 2003 ${ }^{7}$ ). Using Mercury as the reflective liquid is not suitable for a lunar survey telescope to operate in the infrared. The reflecting surface must be much colder than the freezing point of mercury, or it will produce a background thermal radiation much brighter than the very dark natural sky background. The Spitzer telescope shows the extremely deep views that can be reached when this background, set by zodiacal dust, is reached with very cold optics. A key requirement is thus to identify such a very cold liquid, about the temperature of liquid nitrogen, that can be made reflective. The lunar poles are the ideal location, because when shielded from the sun, which is always near the horizon, very low temperatures are reached simply by radiative cooling. The Spitzer and Webb observatories similarly operate in direct sunlight, with their very cold telescopes protected by a radiative shield. The Moon provides a highly favorable environment for cold liquid mirror telescopes, with gravity but unlike Earth with no atmosphere or wind and very little seismic disturbance.

The features of a $20-100 \mathrm{~m}$ survey telescope, operated at a temperature of $\sim 80 \mathrm{~K}$ at a lunar polar location, are illustrated by the artist's impression (Figure 1). The dish of reflecting liquid we envisage to be suspended from a superconducting levitation bearing. The secondary mirror (shown schematically as a $5 \mathrm{~m}$ disc) and the surface deposition equipment are 
supported near the prime focus some $25 \mathrm{~m}$ above the dish. The corrected field is relayed through a central hole in the bearing to an instrument module below, shielded from radiation by lunar soil. Three stations around the perimeter would house magnetic drive and stabilization units, and liquid filling apparatus. The telescope is surrounded by a cylindrical solar radiation shield. This would be of very lightweight multi-layer insulation, given the low gravity and absence of wind. The telescope would be built by some combination of astronauts and robots, taking advantage of capabilities developed as part of future lunar exploration - although it may prove most efficient to rely primarily on robots and telerobotic capabilities

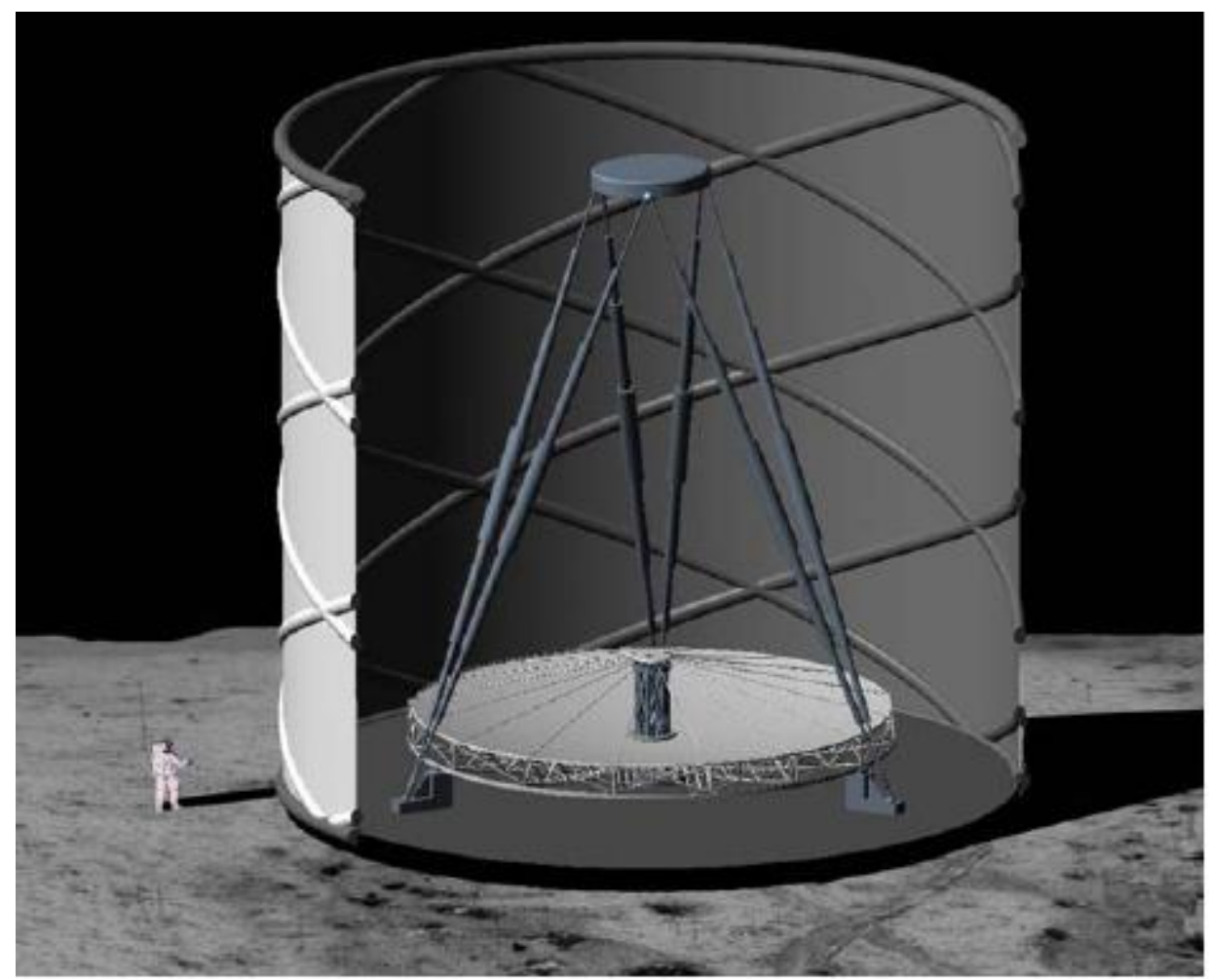

Fig 1. Artist's impression of the $20 \mathrm{~m}$ telescope. The secondary mirror is erected by extending the six telescoping legs, and the sunshield by inflation. The scientific instruments are below the bearing pier, shielded by lunar soil. (drawn by Tom Connors, University of Arizona).

\subsection{SCIENTIFIC VALUE}

We recognize that an enormous investment would be needed to realize a large survey telescope on the moon, justifiable only if the scientific reward were commensurate. Even if the LLMT were considerable lower cost than a similar aperture conventional telescope its location on the moon would make it a major expense item in future exploration budgets. The scientific impact would have to be comparable to that of the Hubble telescope. It is also the case that without the strong support of the astronomical community, the necessary investment would unlikely to be sustained over the many years needed.

Our initial conclusion is that there is potentially a superb and unique scientific role in the study of the early universe, because of the potential on the moon of a cooled telescope to reach much deeper in the infrared than is possible from the ground, and to be built at a size that would likely be prohibitively expensive if built from precision panels in free space. The infrared in the wavelength range between 1 and 10 microns is an extremely important region of spectrum because all of the optical and UV processes that dominate the light from galaxies are shifted into the infrared when one looks at galaxies in the early Universe. Notably one can study rest-frame optical and near-infrared (NIR) properties of galaxies at $1<\mathrm{z}<6$ and study UV and optical properties of galaxies at $\mathrm{z}>6$, where $\mathrm{z}$ is the object's red shift parameter. Modern computer simulations suggest that the first stars form around $z=20$. These stars are super massive, very hot, and shortlived; they dramatically alter their environment by their ionization fronts and supernovae. Typically one believes that the stars completely disrupt the initial gas cloud in which they form. After this epoch, the Inter-Galactic Medium (IGM) is 
likely heated sufficiently that only significantly more massive objects can overcome the thermal pressure and create an environment for gas cooling, collapse, and star formation. These are the first proto-galaxies. The energetic feedback and heavy element production from these early generations of stars provide a crucial boundary condition for the formation of the later generations of stars.

JWST will likely see early galaxies, but it will not see the first stars and it may not be able to see the early protogalaxies. The additional sensitivity of a much larger lunar telescope is needed. The objects are small such that additional angular resolution above JWST would also be valuable. The first stars radiate near the Eddington luminosity and emit predominantly in the ultraviolet. A 100 solar mass star at $\mathrm{z}=25$ would have a continuum luminosity at 3 microns of roughly 15 picoJanskies. Yet more energy is actually emitted in the Lyman alpha and HeII 1640A lines; spectroscopy might be more efficient. Whether or not the Lyman alpha emission can actually escape the IGM is not presently known, but the HeII 1640A lines would and might reach about 200 picoJanskies $(\mathrm{R}=1000)$. Further in the infrared, investigators could look for Balmer lines. These are realistic goals for the $20 \mathrm{~m}$ telescope. Astronomers could look for globular clusters forming at high red shift and study this process in relation to the formation of proto-galaxies. Given that these objects are physically small (proto-galaxies at $z=10-15$ are expected to be marginally resolved by JWST), the additional angular resolution available to a larger telescope would give an increase in sensitivity above the simple increase in light collection. At $50 \mathrm{~m}$ aperture, the diffraction limit at 2 microns is roughly 10 milli-arc-seconds (MAS), which corresponds to a spatial resolution of no worse than 70 parsecs (pc). This means that one can study rest-frame optical properties of galaxies at $\mathrm{z}=3$ with resolution equivalent to a ground-based telescope looking at galaxies in the nearby Virgo cluster. Integral-field spectroscopy could open detailed dynamical and stellar population studies of high-red shift galaxies. High spatial resolution would also be advantageous for investigating super massive black holes at high red shift. One would separate the nucleus from the stars at resolutions considerably above that of HST. Integral-field spectroscopy would permit spatially resolved line diagnostics. One can see black holes of 100,000 solar masses radiating at their Eddington luminosity out to $\mathrm{z}=30$, thereby tracking the growth of black holes along with the growth of galaxies. Of course, the benefits of an IR-optimized observatory have long been recognized, and the James Webb Space Telescope will provide a superb $6 \mathrm{~m}$-class facility. One must consider how a large lunar telescope could improve upon JWST. A key aspect is that a telescope that cannot point cannot take advantage of the closest/brightest examples of a class of local objects. In other words, for topics involving nearby galaxies, star formation, or planets, JWST will usually be able to find a much closer example than those found in a small field of view and thereby make up for its smaller size. However, for galaxy evolution, one is interested in distant galaxies as a class, and for high red shifts, even modest survey areas can include a large and representative volume. Hence, the focus on high red shift galaxies as the science driver for the LLMT. The one exception to this guideline is that from the south lunar pole the LMC is in the field of view, in which case one could image the closest example of a particular class of galaxy. Strong gravitational lensing provides a way for a telescope such as JWST to achieve the effective spatial resolution and light collection of a larger telescope. This may be acceptable for some applications, but the amount of cosmological volume that one can probe with such techniques is very small, many orders of magnitude less than for the zenith pointed LLMT.

\subsection{LLMT DESIGN}

We considered a series of designs beginning with a 2-m precursor telescope, scaling up to $20 \mathrm{~m}$ and $100 \mathrm{~m}$. The scaling parameters are in table 1. A key consideration is the focal plane instrumentation. A $20 \mathrm{~m}$ LLMT telescope will be able to obtain usable infrared spectra at the same limiting magnitude that the JWST can just detect objects, and can detect objects $10 \mathrm{x}$ fainter. For a $15^{\prime}$ field of view and focal plane array with $18 \mu$ pixels this would result in a $\sim 1.6 \mathrm{~m}$ diameter montage of array detectors. With current technology limits of 2000x2000 arrays we might expect within the next decade that technology will improve to the point where individual arrays might have 4000 pixels on a side - requiring about 120 such chips for imaging. Spectroscopic instruments, which could have a primary mission of following up JWST discoveries would be considerably less demanding on detectors. 
Table 1. Scaling parameters for LLMT designs.

\begin{tabular}{|l|l|l|l|}
\hline Diameter $(\mathrm{m})$ & $2 \mathrm{~m}$ & $20 \mathrm{~m}$ & $100 \mathrm{~m}$ \\
\hline Mirror area $(\mathrm{m} 2)$ & $3 \mathrm{~m} 2$ & $300 \mathrm{~m} 2$ & $7600 \mathrm{~m} 2$ \\
\hline Mirror density & $15 \mathrm{~kg} / \mathrm{m} 2$ & $3.3 \mathrm{~kg} / \mathrm{m} 2$ & $5-10 \mathrm{~kg} / \mathrm{m} 2$ \\
\hline Primary mass (tons) & $0.05 \mathrm{mt}$ & $1 \mathrm{mt}$ & $50 \mathrm{mt}$ \\
\hline Total mass (tons) & $0.5 \mathrm{mt}$ & $3 \mathrm{mt}$ & $100 \mathrm{mt}$ \\
\hline Field & $3.1^{\circ}$ annulus & $15^{\prime}$ & 3 \\
\hline Diffraction limit @ $1 \mu \mathrm{m}$ & $0.1^{\prime c}$ & $0.01^{\prime}$ & $0.002^{\prime}$ \\
\hline Pixels @ 2 $\mu \mathrm{m}$ (Nyquist) & $18,000 /{ }^{\circ}$ & 45,000 & 45,000 \\
\hline
\end{tabular}

\subsection{Optical Design}

We have examined a number of design considerations for a LLMT. These results follow directly from the work of Hickson at the University of British Columbia whose $6 \mathrm{~m}$ liquid mirror telescope has demonstrated seeing-limited imaging performance. The lunar telescope is considerably more challenging because it must be diffraction limited. As part of the study, we have explored off-axis systems that would allow increased sky cover. These however generally suffer from field distortion, typically at the level of a percent or so. But once it exceeds the reciprocal of ten times the number of resolution elements across the field $\left(\sim 10^{4}\right)$, it results in unacceptable image degradation during long exposures. This precludes drift-scanning to accommodate star motion away from the zenith, or any kind of long exposures except for the special case when the telescope is located exactly at the Lunar pole or otherwise set to image only a set zenith position, so that a rotation about the symmetry axis of the primary mirror can be made to compensate for Lunar rotation. Designs to circumvent this problem exist but require complex optics and control systems. We conclude that off-axis LLMT's are not practical. A large LLMT should therefore have simple optics that view the zenith. Large reflecting or refracting elements are difficult to support and align. The prospect of accurately deploying, or assembling, a large complex corrector at the top of a $\sim 30 \mathrm{~m}$ high structure on the Moon is daunting. For this reason a Cassegrain type system, with a single mirror deployed above the primary, would be preferred. An example of one such design, with three reflecting mirrors is shown in (figure 2). This design achieves the goal of a 15-arcminute field with diffraction limited images $\lambda \geq 1 \mu \mathrm{m}$ wavelength. The design has an $\mathrm{f} / 1.520 \mathrm{~m}$ parabolic liquid primary, has a $2.4 \mathrm{~m}$ Cassegrain secondary and a $4 \mathrm{~m}$ tertiary mirror. The final focus is at $\mathrm{f} / 15$, for a field diameter of $1.3 \mathrm{~m}$. The imaging array would be a mosaic of $18 \times 18$ four-megapixel arrays, with each 18 micron pixel corresponding to 12.4 milliarcseconds. This provides Nyquist sampling of images down to $1.6 \mu \mathrm{m}$ wavelength. This first optical concept has significant vignetting by the focal plane, and options to reduce this are being explored. This type of design is expandable to larger sizes - perhaps as large as $100 \mathrm{~m}$ diameter.

The distortion in this design is shown for the 15 arc minute field in figure 5 exaggerated by a factor of 100 . For a telescope located precisely at the pole, rotation of the star field about the optical system axis would be exactly compensated for long exposures by rotation of the detector. However, if the telescope were only 7 arc minutes $(3.5 \mathrm{~km})$ from the pole, the star field and the detector would rotate about one edge of the telescope field. The smear caused by distortion for the above design would be about 1 pixel/hour, about the limit we could accept. 


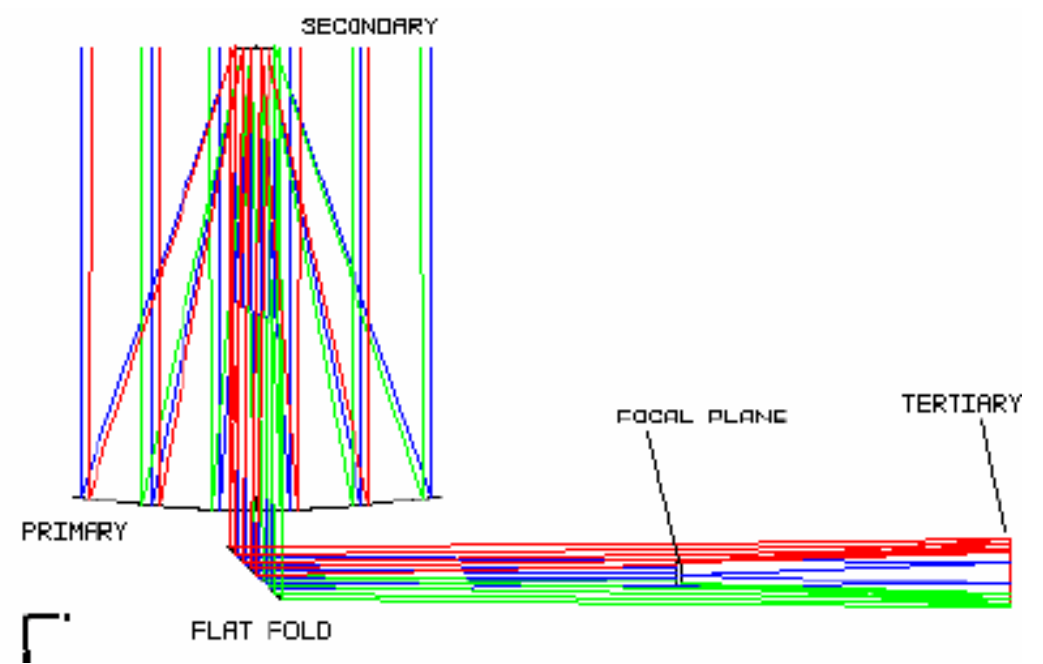

Fig 2. Three mirror telescope design by Dr. Simon Thibault.

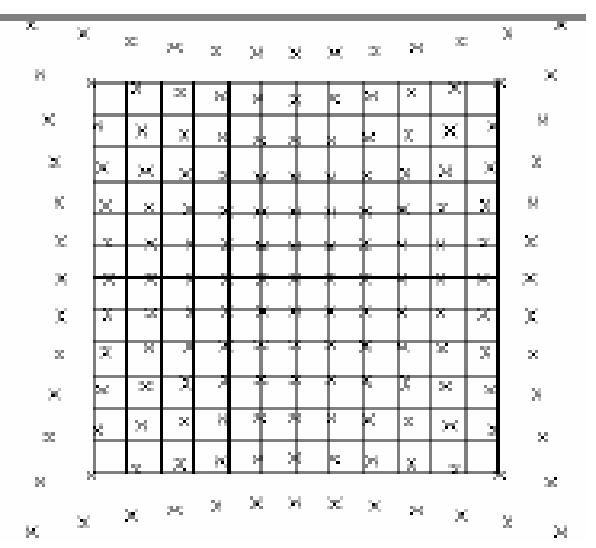

Fig 3. Image distortion for the 3-mirror design shown in Figure 4.

\subsection{Mechanical Design}

A LLMT provides many mechanical design challenges. One needs a lightweight structure that has high stiffness and good thermal stability. For the primary mirror dish, lightweight honeycomb sandwich panels of graphite-epoxy is probably best. This could be made in segments and assembled by astronauts or robots. A space-frame substructure would provide global rigidity and stability. A very important issue is the dynamic control of the primary mirror. Deformations of the primary mirror and support structure result in redistribution of the liquid on the surface, which changes the loading. If the gravitational potential energy released by the fluid exceeds the elastic energy of the deformation, the mirror will be unstable. The softest mode is usually a global tilt resulting from inadequate stiffness in the bearing and support structure. However, the stiffness required to maintain liquid mirror stability for a $20 \mathrm{~m}$ diameter system seems well within current technology for an actively controlled telescope.

\subsection{Superconducting Bearings}

A superconducting bearing for the spinning mirror is an ideal choice, because no lubricant is required and there is a large gap that avoids issues of dust contamination. Ki Bui Ma conducted a series of experiments at the University of Houston with "toy" magnetic suspension bearings. Figure 4 shows the experimental setup and the reflected image in the rotating liquid. The superconductor bearing was an inch in diameter and weighed 85 grams, as listed in table 1 . The spinning dish of liquid weighed $180 \mathrm{~g}$. The speed of rotation ranged from 40 RPM to 60 RPM to yield a mirror of focal ratio f/1. 
The bearing needed to support a 1 ton mass lunar telescope, weighing $160 \mathrm{~kg}$ on the moon and applying this force can be crudely estimated by scaling this design. Since the load capacity increases as the superconductor area and we require a factor of 890 times, from 0.18 to $160 \mathrm{kgf}$, the superconductor diameter must be increased 30 times to $0.75 \mathrm{~m}$. If the scaling is in all three dimensions, the total bearing mass will be 2.4 tons (see table 2). However, the efficiency of mass utilization can be dramatically improved with more sophisticated designs, as demonstrated in smaller versions described in Lee et al (1999) $)^{8}$. An optimized bearing with of 0.3 tons is shown in the third column of the table. . We conclude that a superconducting magnetic levitation bearing is ideal and feasible for a large LLMT telescope. In the model we placed the bearing above the center of mass of the spinning dish, for basic passive stability. The drive torque was by eddy currents in the conducting aluminum mirror dish, induced by a rotating magnet below. In operation, we discovered that the period of rotation settled in to equal the pendulum period of the suspended mirror. The radius of curvature of the liquid surface was then equal to the pendulum length. This has the fortunate consequence that wobble of the mirror at the pendulum period did not upset the natural curvature of the surface, which remains fixed with respect to the dish. . Nature showed how to achieve a naturally stable mirror!

For a diffraction-limited telescope the optical requirements are severe, but by adopting this geometry with the bearing at the center of curvature, we would have a good start. For the spin axis to remain vertical to within $\sim 1$ milliarcseconds, and the gap to be stable to $\sim 1$ micron, to avoid defocus, the required inherent axial stiffness of the bearing is $\sim 40 \mathrm{~N} / \mathrm{mm}$ and the tilt compliance $\sim 0.02 \mathrm{sec}$ of $\operatorname{arc} / \mathrm{mN}^{*} \mathrm{~m}$. These tolerances would be achieved by active control. A telescope mounted on this bearing would have a resonant frequency of around $2.5 \mathrm{~Hz}$. Fortunately, this is considerably higher than the rotation speed of $0.03 \mathrm{~Hz}$. Still, as a vibration isolator, the bearing will not shield the telescope from vibrations below about $3 \mathrm{~Hz}$.

Table 2. Scaling parameters for LMMT superconducting suspension bearings.

\begin{tabular}{|l|l|l|l|}
\hline Model & \multicolumn{2}{|l|}{ Simple scale up } & Optimized design \\
\hline Mirror diameter & $0.2 \mathrm{~m}$ & \multicolumn{2}{c|}{$20 \mathrm{~m}$} \\
\hline Mirror mass & $0.18 \mathrm{~kg}$ & \multicolumn{2}{|c|}{$1000 \mathrm{~kg}$} \\
\hline Mirror weight & $0.18 \mathrm{kgF}$ & \multicolumn{2}{|c|}{$167 \mathrm{kgF}$} \\
\hline YBCO diameter & $25 \mathrm{~mm}$ & $0.75 \mathrm{~m}$ & $1 \mathrm{~m}$ \\
\hline YBCO thickness & $12 \mathrm{~mm}$ & $0.36 \mathrm{~m}$ & $0.025 \mathrm{~m}$ \\
\hline Superconductor mass & $0.055 \mathrm{~kg}$ & 1.53 tons & 0.2 tons \\
\hline Total bearing mass & $0.085 \mathrm{~kg}$ & 2.4 tons & 0.3 tons \\
\hline
\end{tabular}
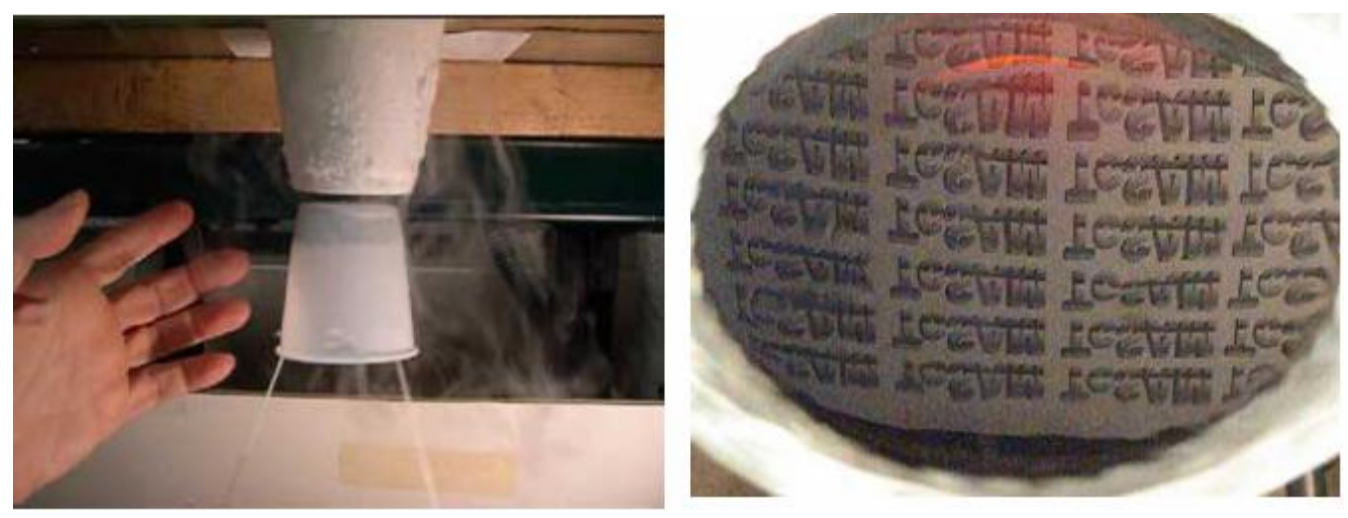

Fig 4. Model of spinning liquid mirror on a superconducting bearing by Ki Bui Ma, University of Houston. (a) shows the bearing, with a nitrogen-cooled YBCO superconductor in the upper cup, and a neodymium magnet in the inverted cup below. The gap is $\sim 3 \mathrm{~mm}$. Hanging on 3 strings below is a $20 \mathrm{~cm}$ spinning dish of black soy sauce with $\sim 8$ " focal length (b). The lettering is the reflection of a screen above 


\subsection{Mirror Liquids and Reflective Coatings}

For thermal emissivity less than the natural zodiacal sky background below 10 microns, the LLMT requires a mirror surface at $<100 \mathrm{~K}$. We need a material that is liquid at this temperature, with very low vapor pressure to prevent evaporation, and either intrinsically reflective or able be metallized to become so. We considered three main classes of liquids that could be used: eutectics of alkali metals, which have the advantage of high intrinsic reflectivity; lowreflectivity liquids coated with a thin metallic coating produced by sputtering or evaporation; and low-reflectivity liquids coated with self-assembling nanoparticles. We concluded that the most promising approach was to apply metallic coatings to low temperature, low vapor pressure moderate viscosity liquids. The reflectivities of the alkali metals are very high (> 98\%) in the blue and the visible and increase monotonically with increasing wavelength. Thompson $(1985)^{9}$ find emissivities of 0.015 for $\mathrm{K}$ and $\mathrm{Na}$. Eutectic alloys of alkali metals have lower melting temperatures than their components. Super cooling, very common among liquid metals, should allow even lower temperature liquids. The two other techniques require a substrate liquid that has a low melting temperature, ideally $100 \mathrm{~K}$ or less, with a metallic coating. A search of chemical databases shows 1 butene as the most promising candidate, liquid at $90 \mathrm{~K}$ with a vapor pressure of 10-7 torr.

Liquid could potentially be coated by vaporizing a reflective metal, borrowing from techniques used to coat solid mirrors on Earth. Ermanno Borra and Omar Seddiki conducted a number of experiments at Laval University to explore the parameter space in this important area, starting with liquids at room temperature. They reached an important milestone, namely the demonstration with two different techniques that it is possible to deposit, in vacuum, a metal on a liquid. This was done with a sampling of the parameter space using a variety of liquids at hand. They found it possible to coat only some liquids. Therefore, before identifying cryogenic liquids suitable for a lunar liquid mirror telescope, one must determine the physical characteristics needed for successful coating. These coating experiments have shown that boiling during vacuum coating was a major problem for most liquids tried. They began by studying the effect of viscosity because our experiments so far indicate it may play an important role. It appears that if viscosity is too small there is no coating; if viscosity is too high, the liquid outgases too slowly and boils during coating because of radiative heating from the hot electrodes. We successfully coated polypropelyne glycol with vacuum deposited tin and silver (Figure 5). With this success we believe that a suitably chosen eutectic liquid with a metallic coating is the most promising approach for a LLMT.

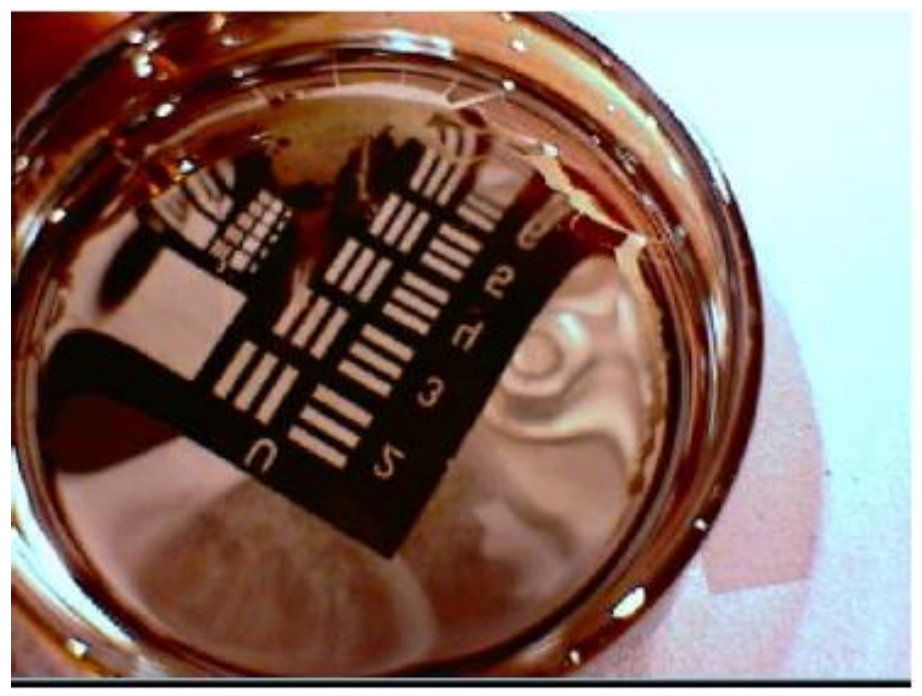

Fig 5. Mirror surface of silver on polypropelene glycol deposited by Ermanno Borra, University of Laval.

\subsection{LUNAR LOCATION ISSUES}

From the previous discussion and experience with large rotating liquid mirror telescopes such as the University of British Columbia's $6 \mathrm{~m}$ system it would appear that liquid mirror technology is potentially a cost-effective approach for very large telescopes in space. Indeed, the key requirement for such a telescope to operate is a reasonable gravity field such as the moons. However, building and operating such a large instrument on the moon presents a unique set of 
challenges and issues (Lester et al 2004) ${ }^{10}$. Whether these issues can be resolved favorable will determine whether our LLMT approach holds any promise.

\subsection{Is Lunar Dust an Issue?}

The possibility has been raised of a "dust atmosphere" above the lunar surface that might increase sky brightness or contaminate telescope optics. Apollo missions have shown the lunar regolith to consist of fine-particulate dust. A literature survey suggests two properties of lunar dust we need to consider:

1. Photoelectric charging of lunar surface by the sun levitates and moves dust near the terminator;

2. A dust atmosphere extending to at least $100 \mathrm{~km}$ in altitude might exist.

We note these properties were derived from only a handful of observations, outlined below, where the existence of levitated dust was only inferred. There has yet to be a direct detection of levitated dust, and there is no knowledge of any levitated dust level at the poles. But the possibility of surface dust levitation to a high enough level to deposit on our optics, and a dust atmosphere could elevate the sky background reducing the sensitivity of our observations has to be considered. Diverse data from the Apollo era suggest the existence of levitated dust near the terminator. Rennilson and Criswell (1973) ${ }^{11}$ modeled the horizon glow observed by Lunar Surveyor 7 Lander a few hours after sunset to be forward-scattered light from a dust layer, consisting of 5-6 micron particles, levitated 10-30 cm in the vertical extent. They suggest the ground and dust are photo-electrically charged by high-energy solar photons creating potential differences between dark and bright regions as the terminator sweeps across the surface. Since the dust is charged, it is transported by electrostatic forces. A robust numerical model for this phenomenon is developed by Pelizzari and Criswell (1978) $)^{12}$. These models are a strong function of solar illumination and only predict low-level dust transport. But due the high obliquity of solar rays in the polar regions, it is possible this would be a negligible effect there. Indeed, a recent paper by Stubbs et al $(2005)^{13}$ proposes that high altitude lunar dust, as discussed below, might be due to solar charging and interaction with the solar wind. In addition, Apollo 15 and Apollo 17 astronaut and Lunokhod-2 observations suggest a completely different phenomenon of a very high, thin dust atmosphere. Apollo 15 astronauts detected an excess of light while photographing the solar corona in orbit just after sunset. McCoy et. $\mathrm{al}^{14}$ (1975) claim this as evidence for a scattering source located a few kilometers above the lunar terminator. While in orbit, Apollo 17 astronauts observed streamers just before sunrise suggesting a scattering atmosphere extending beyond the $100 \mathrm{~km}$ altitude Apollo orbit (McCoy and Criswell 1974 ${ }^{15}$ ). Lastly, the Russian Lander Lunokhod-2 detected unusually bright sky background with a dependence on the zenith angle of the sun, once again suggesting a scattering atmosphere (Severny et. al $1975^{16}$ ). We contacted Apollo 17 astronaut Jack Schmidt, now at the University of Wisconsin, who suggested the following (Schmidt, $2005^{17}$ ):

"The "streamers" we saw appeared to be related to the sun with the moon as an occulting disk of sorts. There is at least one paper on this. I do not believe that these extremely long streamers from the sun out into space were indicators of levitated lunar dust but rather of solar activity. There was some broad horizon glow just before sunrise that may have been caused by dust as seen through a very long path, however, in over 30 years, I understand from the Macdonald Observatory people the corner reflectors have shown no sign of dust accumulation on their cubes. Surveyor saw what some have interpreted as levitated dust at sunset and I think that is in their mission reports. Frankly, I don't think it will be a problem and if it is there may be an electrostatic countermeasure"

We conclude from our preliminary analysis that the presence of dust, either near the lunar surface or suspended at altitude could be a serious, and perhaps decisive problem for a LLMT. We believe that early resolution of this issue in the proposed US space exploration vision should be a high priority.

\subsection{Is star contamination by the Large Magellanic Cloud a problem for the South Pole?}

Our initial thoughts were that the South lunar pole might be ideal for a LLMT. However, courtesy of Bernard Foing and Jean-Luc Josset we were able to study the first ever winter images of the North lunar Pole. Solar illumination near the pole shows it is a viable alternative. This data suggests that the North Pole could be a favorable location for a LLMT. In order to determine the optimum locations we studied the differences in scientific potential of the two poles. Of potential concern is the fact that a zenith pointing LLMT at or near the South Pole would have images contaminated to a degree with stars in the Large Magellanic Cloud. Since the LLMT is designed to carry out long integrations on a small field, a dense, nearby stellar population in the field-of-view can cause confusion problems and reduce the usable field-of-view of 
the telescope. A telescope located at the South Pole will inevitably see part of the Large Magellanic Cloud (LMC), as noted above. A quick look at the Digital Sky Survey (DSS) R-band plates of the North and South Pole fields, shown in Figure 6 illustrates the potential problem. At an R-band limiting magnitude of 21 there is significant stellar contamination in the South Pole field, though the angular resolution is only 2". At our proposed LLMT sensitivity, we will reach a $5 \mu \mathrm{m}$ wavelength limit of 26 mag with 0.05 " resolution, comparable to Hubble's red-end resolution. This means we will see all of the Main Sequence (MS) stars of the LMC and reach about a magnitude fainter. This may not be an issue if our telescope has sufficiently high angular resolution to resolve out the stars at our sensitivities. The North Pole location, as shown by the contrast in stellar densities depicted in Figure 6, does not experience this problem as it looks well away from the galactic plane with no nearby galaxies in the field of view.

To assess the South Pole site carefully, we need an estimate of the surface density of LMC stars to place constraints on the size of a usable field. We address this by modeling the LMC stellar population using STARFISH (Harris et al, $2001^{18}$ ), a code that generates a stellar population and associated photometric values for each star from a specified star formation history (SFH). Using a built-in SFH derived specifically for the LMC, STARFISH generates results that match the observations of a complete LMC stellar catalogue, (Zaritsky et al, $2004^{19}$ ) which samples the bright and "giant"-end of the stellar distribution. We extend the model to the faint-end of the MS to get a crude idea of expected stellar surface densities at our sensitivity. We use the catalogue stars within a 5' field of the south ecliptic pole (SEP) to be representative of the top end of the stellar population (Vlimit $=22$ ) and derive a stellar density of 0.005 stars/arcsec2. We scale our simulation accordingly to obtain a predicted LMC stellar density of 0.3 stars/arcsec2 down to a Vlimit of 33 , corresponding to the faintest MS stars in the LMC. At the M-band diffraction limit, we would expect about $99.9 \%$ of field to be usable. To add credence to this analysis, we performed the same analysis using an archival Hubble ACS High Resolution Camera (HRC) F606W image of a field close to the South Ecliptic Pole. This exposure is deeper than the ground-based data and is able to detect V=26 stars in the LMC at 4-sigma. Unlike the DSS observations HST resolves all of the stars. Our simulations predict the number of stars observed in the HST image within a factor of three. If we use the HST-derived surface density for our top-end calibration, we derive a predicted LLMT-observed surface density of 0.7 stars/arcsec 2 . This translates to a $99.8 \%$ usable field in the M-band. The main sources of uncertainty in this analysis arise from the imprecise determination of the LMC SFH, and the luminosity distribution at the faint, low mass end. However, we would require 50 times as many sources as our predictions to decrease our usable field-of-view to $90 \%$. Such a large discrepancy from our modeling seems unlikely, which leads to our conclusion that LMC stars will only be a minor interference to a South Pole LLMT. We thus conclude that either pole would be suitable for the primary scientific objectives of an LLMT. Decisions as to which Pole is optimal would thus be determined by other factors such as potential science payoff from secondary objectives as studying stars within the LMC and logistics factors as availability to power systems and distance from interference.

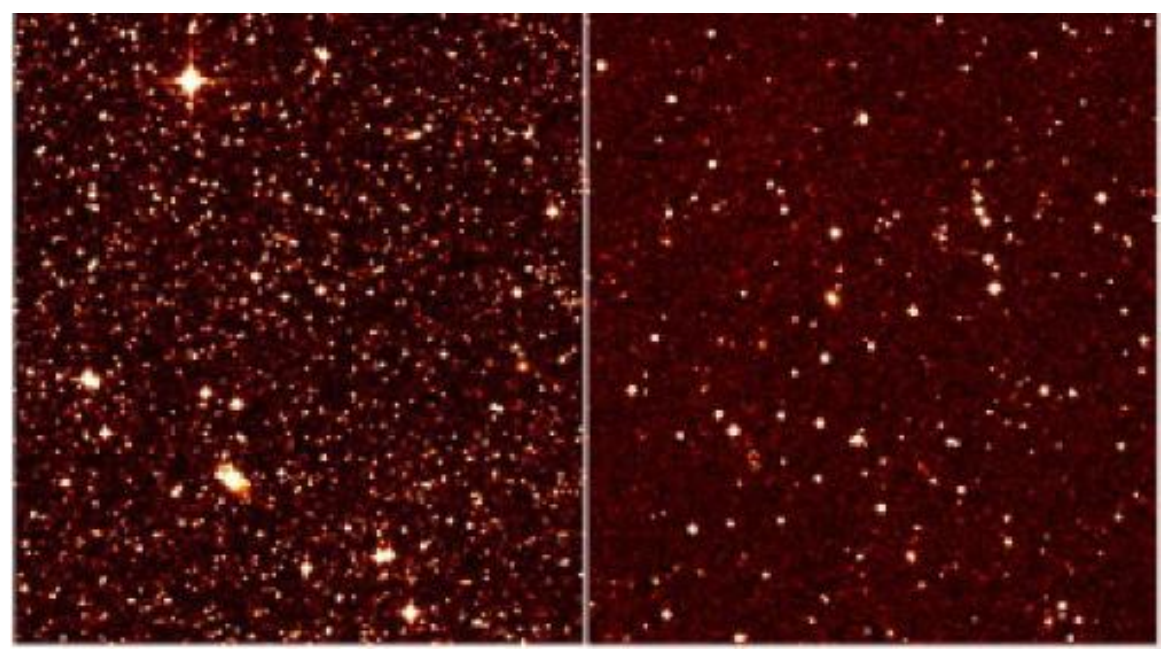

Fig 6. Comparison of N and S ecliptic poles. Schmidt survey photographic R-band images; Left - South Pole view, Right North Pole view. $\mathrm{R}_{\text {limit }}=21 ; \mathrm{FWHM}=2$ '; FOV $=12$ ' x 12'. 


\subsection{Sky Coverage and Distance from the Lunar Pole}

We consider the case of a zenith pointing telescope with 15 arc minute field. If it is placed directly at the pole, the observed field rotates around its center once per month, at a center 1.55 degree off the ecliptic pole. Over the course of the 18.6 year lunar precession cycle, the view will trace a circle around the ecliptic pole. The result would be to sweep out an annulus 9.7 degrees in circumference and $15^{\prime}$ in w centered on the ecliptic pole, dwelling on individual objects continuously for about 6 months. If the telescope is off the pole, the view will sweep around in a circle once per month, and the center of the will slowly move around the ecliptic pole. In 18 years, 2 square degrees would be covered with integration time close to 6 months for all points. An alternate location of especial interest would be at 1.55 degrees latitude from the lunar pole. Here the telescope and detector suite would sweep out a 9.7 degree annulus once each lunar month centered on the lunar pole position, but passing through the ecliptic pole monthly for about 1 day a month . Over 18 years this would provide a 6 month total integration on a 15' square image of the ecliptic pole, dropping off the typically 3 weeks over a 30 square degree area.
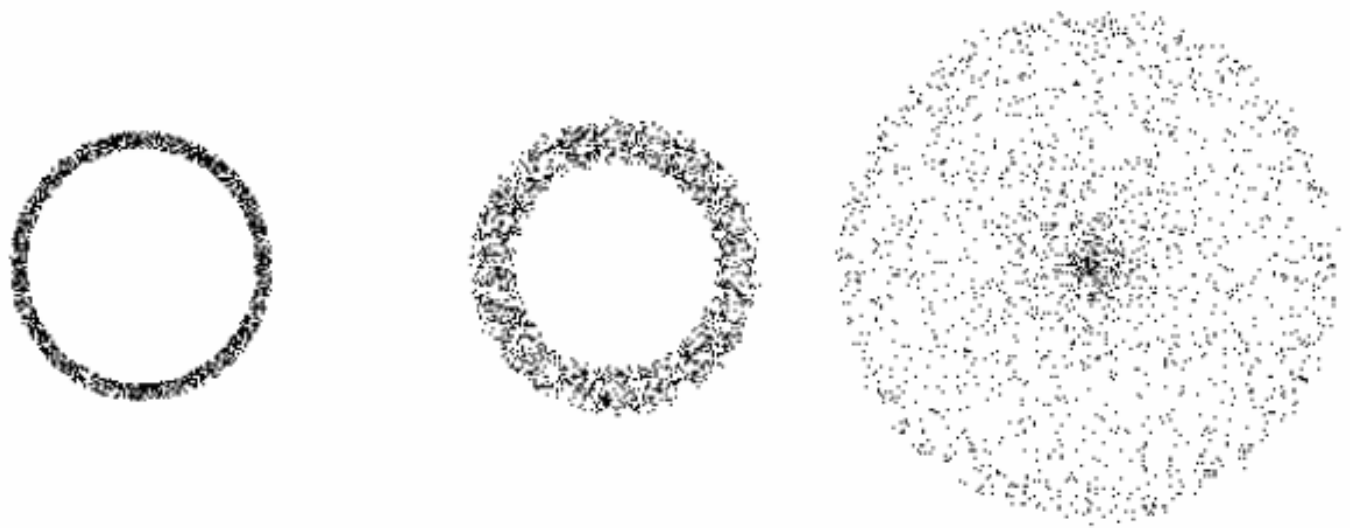

Fig 7. Effect of location on sky access for a zenith-pointed telescope with 0.2 degree field of view. The integrated exposure over 18 years is shown in each case. Left - at the pole, over 18.6 years the field sweeps out an annulus 3.1 degrees in diameter centered on the ecliptic pole, with continuous integration of $\sim 5$ months on any one spot. Center -0.2 degrees from the pole, the field sweeps out a half degree annulus each month, covering any one spot every month for a year Right -1.55 degrees from the pole. Each month the field sweeps a 3.1 degree annulus, covering any spot for about 15 hours. The ecliptic pole is seen for this time every month, for a total integration time of 5 months over 18 years.

\subsection{Solar Power and Other Location Considerations}

The precise location for an LLMT depends on both scientific and non-scientific factors. For example, to place a telescope exactly at the South lunar pole would place it within the permanently shaded Shackleton Crater (see Bussey et al., $1999^{20}$ ). This location could have the advantage of very low ambient temperature and minimal possibility for problems from solar levitated dust. However, this location would be difficult to access for construction and maintenance and would require large amounts of beamed power as well as complicated data relay systems as it is permanently out of the line of sight from the Earth. Moreover, this crater could become the scene of volatile extraction operations that could produce vibrations, dust and other interference with astronomical observations. Alternate locations for an LLMT would be in locations with near continuous solar illumination - so called "peaks of eternal light." One such site is the rim of the Shackleton Crater. These locations have the potential advantage of easy access to solar power during most of the lunar day-night cycle, benign thermal conditions and potential frequent direct access to the earth for communications.

Based on our conclusion that the North lunar pole offers a deep view less contaminated by faint stars, we investigated its suitability for LLMT sites. A good study of lunar polar illumination was made from the 1994 Clementine data, which was orbiting at a time of winter at the south pole and summer in the north. A recent Nature article by Bussey et al $(2005)^{21}$ identifies permanently illuminated locations during summer at the North Pole. However, until now there have been no observations of the North pole in winter. Fortunately, the ESA SMART-1 probe arrived at the moon during our Phase I work. In a collaborative effort with ESA's lunar orbiter team, we obtained the first images of the North Pole in winter. SMART-1 arrived at the Moon in November 2004 and has since spiraled down to a polar orbit. During the 
commissioning period and between orbit modifications, the orbiter imaged strips of Moon with its $5^{\circ} \times 5^{\circ}$ AMIE camera. We analyzed available images of the North Pole region (hereafter NPR) acquired during that period, which was close to the January 25th 2005 winter solstice. Our preliminary analysis shows ridges and crater rims within $0.5^{\circ}$ of the North Pole are illuminated for at least some sun angles during lunar winter. The SMART-1 images indicate that solar power for a polar telescope could be obtained even in winter from locations within $15-20 \mathrm{~km}$ from the true pole. The North Pole lies in a lunar highlands area and appears to have to peaks within $\sim 20 \mathrm{~km}$ of the pole that between them even in winter could provide permanent solar power transmitted either through cables or beamed via microwave links. The SMART-1 data from Jan 192005 reveals an illuminated peak near the pole corresponding to one of the permanently sunlit areas in the 1994 Clementine summer data (Foing, et al, 2003 ${ }^{22}$ ). Additional data from SMART-1 during 2005-6 will help refine thee results by providing a complete illumination map of the polar region for the entire year. This will enable us to determine the best locations - both at the pole and at 1.55 degrees to access the ecliptic pole. The data to date suggests that a LLMT might be built during the lunar summer using natural illumination. As noted above we believe there are two viable modes of operating a LLMT. One mode would obtain very deep images of an annulus centered on the pole and covering slightly more than one square degree. A second mode would place the LLMT at a location 1.55 degrees from the pole pointed at the ecliptic pole. This would produce a "hula-hoop" pattern on the sky with only a single region at the ecliptic pole having very deep images. However, this small patch of sky would be repeatedly imaged once each lunar month enabling synoptic observations within this region. And the image from this 15 arc minute field would be just as deep as for the larger area in the alternate scheme covered during the 18.6 year observing run - albeit with much less deep integration times. Since $\mathrm{z}=10$ objects have a time dilation where transient events observed from earth appear to evolve 10x slower, interesting scientific opportunities may exist. For example, supernovae in the early universe could be followed even allowing for time dilation by a factor of 10 . The scientific advantages and regrets from these two modes will need to be fully investigated.

\subsection{PRECURSOR MISSIONS}

While the data we have obtained to date suggests the North lunar pole offers superior benefits it remains to determine a specific set of locations upon which to focus future efforts. Understanding the scientific factors will be important if, for example, plans are made to dedicate one pole to intensive development activities that would generate dust, and the other to quieter scientific activities. Existing Clementine data and ESA SMART-1 data offers us an opportunity to obtain the necessary information to fully characterize a range of potential sites. Within this trade must be a determination of whether sites at or near the pole or 1.55 degrees off the pole are more scientifically advantageous. We believe a low cost lunar Lander will be needed to resolve the over-riding issue of lunar dust.

It does not appear possible to resolve without more data the issue of whether naturally levitated dust will be a factor at the poles. In addition, line of sight views of earth and the sun will be needed to definitively select a suitable site. An in situ site survey covering summer and winter is therefore needed. A site survey mission should have several key capabilities. It would need to determine whether the sky background is raised by a dust atmosphere. This would be done in the infrared and visible bands for at least a year to cover both lunar winter and summer. In addition, it would be placed right at the best candidate site to directly measure the horizon location, and possible obstacles relative to the sun for the same period. In addition it would need to determine whether the ambient dust environment presents a serious threat to optical, particularly liquid surfaces. At the end of the one year mission, we would measure the result of simulated human and robotic activities at various distances to see what level of activity is acceptable before dust on the optics becomes a problem. We have sketched what a simple instrument package might look like in Figure 8. We envisage a payload mass of no more than $10 \mathrm{~kg}$. To be practical such a site survey must be light weight and represent an affordable mission. Millennium Space of El Segundo, CA has designed lunar Lander missions with estimated costs of \$50M US. We believe such a low cost approach is a critical next step. in obtaining the necessary data and support to further develop the LLMT concept.

Assuming successful site survey missions an intermediate step could be to construct and deploy automatically on the lunar pole a $2 \mathrm{~m}$ class LLMT which could undertake an already unique deep survey and serve as an engineering validation. We have produced preliminary designs for a 1.7 meter wide field telescope with diffraction limited resolution 0.3 arcsec at 2.5 microns wavelength. This system has a 3 degree annular field, 14 minute wide which would be fully covered by a ring of forty 4096 square detector arrays with 0.2 arcsec pixels. The different detectors would have 
different filters in front (like the present Sloan digital sky survey camera), so as to provide up to 40 wavelength bands. Its limiting sensitivity in any one band would be some 25 times deeper than the Spitzer Telescope in the same band i.e. $20 \mathrm{nJy}$ at $3.5 \mu \mathrm{m}$. Located 1.55 degrees from the pole, it would provide a deep finder survey that could be of great value in optimizing the JWST program, if it were in place at the same time. Our preliminary analysis shown in Table 1 suggests a relatively low weight and limited complexity $2 \mathrm{~m}$ system is possible. Such a test and validation mission could easily be part of proposed NASA sortie missions to one of the lunar poles late next decade.

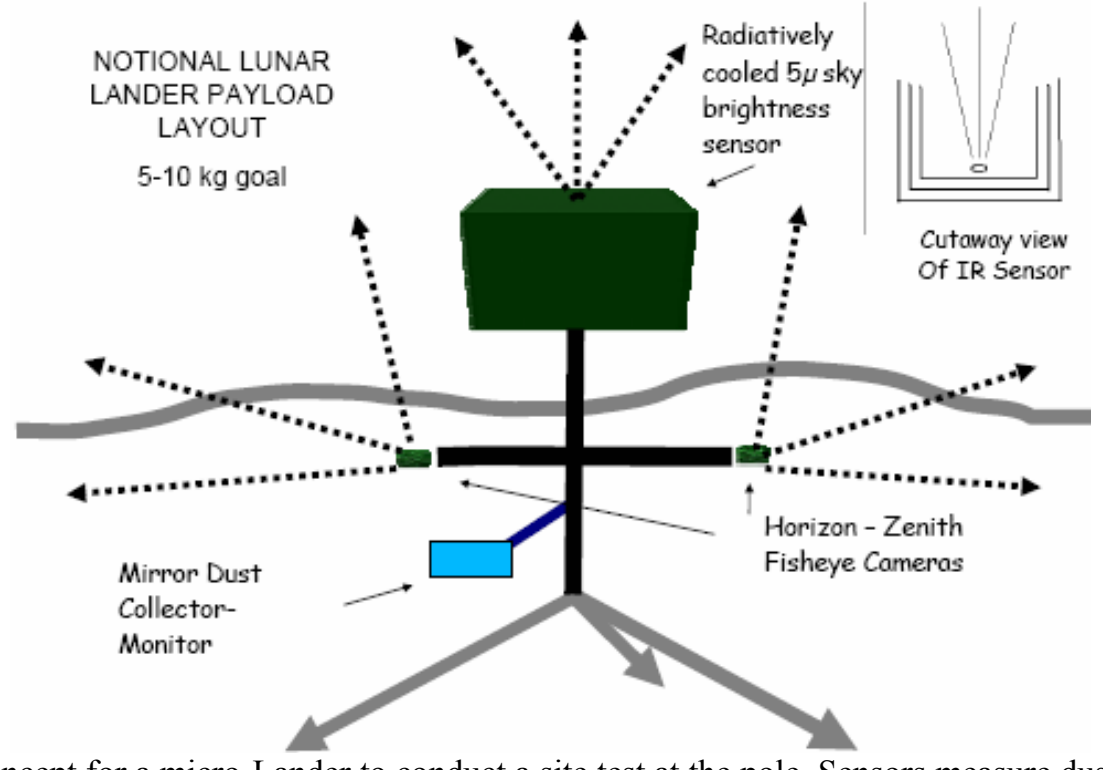

Fig 8. Design concept for a micro-Lander to conduct a site test at the pole. Sensors measure dust deposition and any thermal emission from a dust atmosphere above. Cameras view the terrain and sun around the horizon.

\subsection{SUMMARY AND CONCLUSIONS}

We have proposed a novel Lunar Liquid Mirror Telescope (LLMT) concept. Making use of lunar gravity we believe a rotating liquid mirror telescope of 20-100m diameter might be affordably constructed at one of the lunar poles. Such an instrument could provide unique insights into the early universe - at epochs prior to galaxy formation during which the first stars were formed, providing important constraints on current fundamental astrophysical and cosmological issues such as the origin and nature of dark matter and dark energy.

Technology appears available to make such a telescope feasible and affordable considerably sooner than would be possible for a free-flyer of similar aperture. However, a LLMT has significant limitations. It only points at a limited annulus near the lunar pole and is not suitable for general purpose astronomical investigations of the type possible with free-flying observatories such as the James Webb and Hubble Space Telescopes. These limitations and the likely significant costs argue for an in-depth analysis and debate within the astronomical community trading costs versus scientific benefits. Moreover, the feasibility of a LLMT depends critically upon the level of infrastructure and support from the proposed space exploration vision. For example a $2 \mathrm{~m}$ diameter scaled LLMT could be accomplished as an early part of proposed lunar sortie missions late next decade. While having LLMT as a part of this exploration vision could enhance its likelihood and make it affordable, having such a major scientific mission closely tied to high priority human exploration could also threaten its success and even limit other high priority scientific missions not so closely tied to human activities. These issues must be fully examined as part of the scientific dialogue we propose to begin now. There may be significant technical limitations with our LLMT concept. We have not yet identified a suitable liquid for low-temperature operations on the lunar surface, but are optimistic such a material can be found or developed. More significantly there remain potential show-stopping problems with lunar dust. If measurable quantities of lunar dust are suspended at distances of tens of meters to hundreds of kilometers above the lunar surface the scattered light from this material could eliminate any advantage a LLMT might have for investigating the early universe. We propose an early low-cost lunar robotic Lander mission to determine whether these problems exist. In addition such a Lander could find and characterize the optimal location for a LLMT. The possibilities of interference from other human and robotic activities on the moon is also a concern, albeit one that might be solved with appropriately-selected thin liquid films 
and/or viscous liquids. Another solution might be to designate one lunar pole a scientific reservation free from large scale activities such as in-situ resource utilization. Despite the issues and limitations we believe that a LLMT should be a high priority possibility for future lunar activities. The potential scientific payoff appears significant and appealing.

\section{ACKNOWLEDGEMENTS}

The authors would like to thank the NASA Institute for Advanced Concepts and the Canadian Space Agency for supporting the initial concept work for a LLMT.

\section{REFERENCES}

1. Girard, L., Borra, E.F. 1997, ApOp., 36, 6278.

2. Tremblay, G., and Borra, E. F., 2000, ApOp, 39, 3651.

3. Sica, R. J., Sargoytchev, S., Borra, E.F., Girard, Argall, S., Sarrow, C.T., \& Flatt, S. 1995, Appl. Opt., 34, 6925.

4. Hickson, P., \& Mulrooney, M. 1998, ApJ Suppl., 115, 35.

5. Cabanac, Rémi A. Borra, Ermanno F., Beauchemin, Mario, 1998, ApJ, 509, 309.

6. Cabanac, R. A., Hickson, P. and de Lapparent, V. 2002, "The Large Zenith Telescope Survey: A Deep Survey Using a 6-m Liquid Mirror Telescope," in A New Era in Cosmology, eds Metcalfe, N. and Shanks, T. ASP Conference Proceedings 283. p 129.

7. Hickson, P., and Lanzetta, K.M. 2003, "Large Aperture Mirror Array - conceptual design for a distributedaperture 41-meter telescope," in Future Giant Telescopes. Edited by Angel, J. Roger P.; Gilmozzi, Roberto. Proceedings of the SPIE, Volume 4840, pp. 273-282.

8. Lee, E.K., K.B. Ma, T.L. Wilson, and Wei-Kan Chu 1999, "Characterization of Superconducting Bearings for Lunar Telescopes," IEEE Trans. On Applied Superconductivity, 9, 911.

9. Thompson, J.C, 1985, Handbook of Thermodynamics and Transport Properties of Alkali Metals.

10. Lester, Daniel F., Yorke, Harold, W., and Mather, John C. 2004, Space Policy, $20,99$.

11. Rennilson, J. J., and Criswell, D. R. 1974, The Moon, 10, 121.

12. Pelizzari, M. A., and Criswell, D. R. 1978, LPSC, 9, 3225.

13. Stubbs, T.J., Vondrak. R.R. and Farrell, W.M., 2005, Lunar and Planetary Science, 36, 1899.

14. McCoy, J. E., Ross, C. L. and Criswell, D.R . 1975, LPI, 6, 537.

15. McCoy, J. E., and Criswell, D. R. 1974, LPI, 5, 475.

16. Severny, E. I. T., Zvereva, A. M. 1975, The Moon, 14, 123.

17. Schmitt, Harrison H., 2005, private communication.

18. Harris, J. and Zaritsky, D. 2001, ApJ Supp. 136, 25.

19. Zaritsky, D. Harris, J., Thompson, I. B., and Grebel, E.K. 2004, AJ, 128, 1606.

21. Bussey, D.B.J., Fristad, Kirsten E. Schenk, Paul M. Robinson, Mark S. and Spudis, Paul D. 005, Nature, 434, 842 .

20. Bussey, D.B.J., Spudis, P.D., and Robinson, M.S., 1999, Geophys Res. Lett. 26, 1187-1190.

22. Foing, B.H., et al 2003 , , "SMART-1 Mission to the Moon: Technology and Science Goals," Advances in Space Research, 31, 2323. 